Arch Phys Med Rehabil. Author manuscript; available in PMC 2013 March 12.

Published in final edited form as:

Arch Phys Med Rehabil. 2008 March ; 89(3): 538-542. doi:10.1016/j.apmr.2007.11.009.

\title{
Measuring Fatigue in Persons with Spinal Cord Injury
}

Hubert A. Anton, MD, FRCPC ${ }^{1,3,4}$, William C. Miller, Ph.D., OT OT $^{2,3,4}$, and Andrea F. Townson, MD, FRCPC $1,3,4$

${ }^{1}$ Division of Physical Medicine and Rehabilitation, University of British Columbia

${ }^{2}$ School of Rehabilitation Sciences, University of British Columbia

${ }^{3}$ International Collaboration for Repair Discovery

${ }^{4}$ Vancouver Coastal Health Research Institute

\section{Abstract}

Objective-To evaluate the psychometric properties of the Fatigue Severity Scale (FSS) in persons with spinal cord injury (SCI).

Design-A two week methodological study was conducted to assess the internal consistency, reliability and the construct validity of the FSS.

Setting-A tertiary spinal cord rehabilitation facility.

Participants-48 community living individuals at least one year post SCI with ASIA A or B SCI and no medical conditions causing fatigue.

Main Outcome Measures-The ASIA Impairment Scale; the FSS; a Visual Analogue Scale for Fatigue (VAS-F), the SF-36 vitality scale, and the Centre for Epidemiological Studies Depression - Scale (CES-D)

Results-Our sample was predominantly male $(\mathrm{n}=31,65 \%)$ with tetraplegia $(\mathrm{n}=26,54 \%)$ and ASIA A injuries $(\mathrm{n}=30,63 \%)$. The mean FSS score at baseline was $4.4(\mathrm{SD}=1.4)$ with $54 \%$ $(\mathrm{N}=26)$ scoring greater than 4. The internal consistency of the FSS was Cronbach's alpha $=0.89$. Two-week test-retest reliability was ICC $=0.84(95 \%$ CI $0.74-0.90)$. The magnitude of the relationship was as hypothesized for the VAS-F(r=.67) and CES-D ( $\mathrm{r}=.58)$ and lower than hypothesized for the vitality subscore $(r=-.48)$ of the SF-36.

Conclusions-The FSS has acceptable reliability with regard to internal consistency, test-retest reliability, and validity in persons with motor complete SCI.

\section{Keywords}

fatigue; spinal cord injuries; rehabilitation

Please address correspondence to: H. A. Anton, MD, Pacific Rehabilitation Medicine, Suite 140- 943 West Broadway, Vancouver BC, V5Z 4E1, Telephone 604-733-2222, Fax 604-733-2202, hanton@ pacificrehab.net.

Reprints will not be available from the authors 


\section{INTRODUCTION}

Fatigue is a universal human experience that can negatively affect participation in daily tasks, recreation, and work. The documented prevalence of fatigue is as high as $90 \%$ in certain groups ${ }^{1}$. Fatigue is common in multiple sclerosis (MS), stroke, polyneuropathy, and poliomyelitis. ${ }^{2,3,4,5}$ In persons who are already dealing with significant neurologic or musculoskeletal impairment the effects of fatigue on function may create an additional barrier to community reintegration.

It has been our clinical experience that fatigue is a common problem in persons living with spinal cord injury (SCI). The literature suggests as many as $25 \%$ of persons living with the late effects of SCI report fatigue severe enough to affect their function or qualify of life but the true prevalence of fatigue in that population is unknown. ${ }^{6}$ Fatigue may become more significant with aging in SCI. ${ }^{6,7,8} \mathrm{SCI}$ is associated with many secondary medical conditions that could contribute to fatigue, including anemia, depression, autonomic dysfunction, and chronic pain. ${ }^{9,10,11,12,13}$ Other possible causes of fatigue include deconditioning due to reduced mobility and the increased energy costs associated with living and functioning with a physical disability. ${ }^{14}$

There are important gaps in our understanding of fatigue after SCI. More information is required about the true prevalence, magnitude, and significance of the problem. Evidence based strategies are required for persons living with SCI to manage their fatigue and maximize their function. A number of tools are currently used for measuring fatigue in populations with neurologic disorders. ${ }^{3,4,5,15,16} \mathrm{We}$ are aware of no validated measures of fatigue for persons with SCI. Clinicians and researchers require valid and clinically useful tools to measure fatigue, to assess the outcomes of treatment for fatigue, and to support high quality research in fatigue associated with SCI.

The Fatigue Severity Scale (FSS) is a measure originally developed for use in MS. ${ }^{15}$ It has been extensively validated in other settings and may be the most widely used measure of fatigue in neurologic disorders. ${ }^{4,5,15,16,17,18,19,20,21}$ The FSS is easy to use both in clinical practice and research. It was designed to measure both fatigue and the effects of fatigue on function, which makes its use in rehabilitaton settings particularly appealing.

The purpose of this study was to evaluate the psychometric properties of the FSS for assessment of fatigue severity within the SCI population. The specific objectives of the study were to assess the internal consistency, the 2 week test-retest reliability, and the construct validity of the FSS in SCI.

\section{METHODS}

\section{Design/Sample}

We used a two week follow up methodological design to address our study objectives. A consecutive sample of 54 subjects more than one-year post traumatic SCI was recruited from the patient database of the G.F. Strong Rehab Centre in Vancouver, British Columbia. The G.F. Strong Rehab Centre is a tertiary referral centre that provides inpatient and outpatient 
rehabilitation services to residents of the province of British Columbia. It is the main provider of inpatient rehabilitation to persons with traumatic SCI in British Columbia.

Inclusion Criteria were ASIA (American Spinal Injury Association) A or B (motor complete) $\mathrm{SCI}^{22}$; SCI more than one year previously; and residence within $50 \mathrm{~km}$ of the study centre. Exclusion criteria were inability to provide informed consent; inability to read and write English; age under 19; a co-morbid medical such as a neurological or psychiatric condition likely to cause clinically significant fatigue; ventilator dependent tetraplegia; or a significant change in health status in the interval between test and retest.

\section{Measurement}

Data collected on initial assessment included subject demographics and medical information relevant to fatigue. A physician trained in the use of the ASIA assessment system recorded the ASIA Impairment Scale and motor score. The FSS was measured and recorded. Other measures completed included the Visual Analogue Scale for Fatigue (VAS-F) ${ }^{15}$, the vitality scale of the Short Form-36 (SF-36) ${ }^{23,24}$ and the Centre for Epidemiological ScaleDepression (CES-D). ${ }^{25,} 26$ The additional measures were used to determine the validity of the FSS.

The FSS is a 9 item measure of the severity of fatigue (Figure 1). It requires the participant to choose the degree of agreement on a 7 point ordinal scale ranging from 1 (strongly disagree) to 7 (strongly agree). Scores are calculated by deriving an arithmetic mean. Cut scores of over 4 are indicative of significant fatigue ${ }^{15}$. The reliability and validity of this tool in other populations has been reported to be good. ${ }^{5,15,17,18,19,21}$ Initial studies of the FSS to assess test-retest reliability of the FSS in MS and systemic lupus erythematosus (SLE) demonstrated a correlation coefficient of $0.84 .{ }^{15}$ We hypothesized the FSS would have testretest reliability of $>0.80$ as demonstrated by intra-class correlation coefficient (ICC). The internal consistency of the FSS using Cronbach's alpha in MS was 0.81 and in SLE 0.89. ${ }^{15}$ We hypothesized the FSS would have internal consistency of Cronbach's alpha of $\geq 0.9$.

The VAS-F is a simple one item question of self reported fatigue similar to a visual analogue scale for pain. Variants of the VAS-F have been widely used in fatigue research and practice. $5,15,27$ We used a VAS-F with anchors of "no fatigue" and "extreme fatigue". Subjects marked a point somewhere along a $100 \mathrm{~mm}$ line indicating the severity of fatigue. The VAS$\mathrm{F}$ was scored from zero to 10 . The VAS-F has previously been shown to have a significant correlation with the FSS $(r=0.68)$ in MS and SLE and moderate correlation in post polio syndrome $(\mathrm{r}=0.45) .5,15$ We hypothesized the FSS would have a statistically significant positive correlation with the VAS-F of $>0.7$.

The SF-36 is a widely used measure for assessing Health Related Quality of Life that has demonstrated reliability in many settings. ${ }^{23}$ It was designed for use in clinical practice and research to provide information about individual mental and physical health status. Eight health concepts are covered including: 1) limitations in physical activities; 2) limitations in social activities; 3) limitations in usual role activities due to physical and 4) emotional problems; 5)bodily pain; 5) general mental health; 7) vitality (feelings of energy/the absence of fatigue); and 8) general health perceptions. Some parts of the SF-36 are not applicable to 
SCI but there is support for its use in the SCI population. ${ }^{24}$ We chose to compare the FSS to the vitality scale of the SF-36 as it has the greatest relevance to the issue of fatigue. Previous research has shown a strong correlation between the FSS and SF-36 vitality score in chronic hepatitis $\mathrm{C}(\mathrm{r}=-0.76) .{ }^{18}$ We hypothesized the FSS would have a negative correlation with the vitality component of the SF-36 ( $r>-0.7)$.

There is a significant overlap between depression and fatigue. We therefore chose to compare the FSS to a measure of depression. The Centre for Epidemiological Studies Depression - Scale (CES-D) has been used widely in studies of the general population. ${ }^{25}$ The CES-D contains 20 items. Responses are rated on a 3 point scale ranging from 0 (rarely or none of the time) to 3 (most or all of the time). An overall score is calculated by summing the individual item scores. In the SCI population scores over 19 indicate significant or mild depression with a reported sensitivity of $80 \%$ and specificity of $68 \%$. Cronbach's alpha among a diverse sample of individuals with SCI was $0.88 .{ }^{26,28}$ Previous work by Krupp et al showed a correlation with the FSS of 0.2 in controls, 0.26 in subjects with MS, and 0.46 in subjects with SLE. ${ }^{15}$ We hypothesized the FSS would have a positive correlation with the Centre for Epidemiological Studies Depression - Scale (CES-D) of $r>0.5$.

\section{Protocol}

Subjects recruited to the study were provided with a testing time that was convenient to their schedules. Upon arrival at the center all questions regarding the study were addressed and consent was obtained. All measures were then completed and the ASIA assessment was performed by a physician. The entire session took less than 60 minutes. Participants received a $\$ 50$ stipend to cover costs of transportation and their time. The subjects were retested with the FSS 2 weeks later. Most returned to the center to complete the second FSS, but 4 subjects were unable to travel to the center and were allowed to complete the questionnaire at home and return it via surface mail. The 2 week follow-up was chosen as long enough to eliminate the effects of learning and short enough to reduce the likelihood of an interval change in personal or medical situation that could affect fatigue. The subjects were screened on retesting for changes in their situation that could change their fatigue level, including new medical conditions, change in medication, change in caregiver situation, or change in activity. Subjects were excluded from the study if such changes occurred. The study protocol was approved by the Clinical Research Ethics Review Board of the University of British Columbia.

\section{Data Analysis}

Based on the work of Donner and Eliaszew, we calculated a sample size of 42 would provide sufficient power to assess our hypothesis that the reliability of the FSS would exceed an ICC $>0.80$ given alpha of .05 and beta of .80 . Descriptive statistics such as means, standard deviations and proportions were used to provide a summary of all variables. Cronbach's alpha was derived to assess reliability related to internal consistency and one-way analysis of variance (ANOVA). Interclass Correlation Coefficients (ICC 1,1) were calculated to assess the two week reliability of the total score and the item by item scores of the FSS. The standard error of measurement (SEM) was used to assess how an individual score varied on repeated measurement. ${ }^{30}$ The SEM was calculated by multiplying the standard deviation by 
the square root of 1 minus the reliability coefficient (ICC). The Bland-Altman (or Limits of Agreement) method was also used to provide an assessment of absolute agreement between the baseline and follow up ratings and to identify any bias that might exist. ${ }^{31}$ The BlandAltman method provides a visual representation that is easy to understand and interpret. ${ }^{32}$ The difference in FSS scores between the two data collection sessions is plotted against the mean score for each subject. The mean and standard deviation of the mean difference as well as the true value of the mean using $95 \%$ confidence intervals (CIs) were calculated to further assess the existence of bias.

Analysis of validity was performed using Pearson's product moment correlation. We also assessed the sensitivity and specificity values and the associated $95 \%$ confidence intervals of the FSS. Based on previous work, we assumed a cut score of greater than 4 on the FSS indicated significant fatigue ${ }^{15}$ but we also provide values for a score of 5 . There is no gold standard of fatigue measurement in SCI but we chose a score of greater than 6 (out of 10) on the VAS-F to indicate severe fatigue. That allowed us to create a receiver operating characteristic (ROC) curve and calculate the area under the curve (AUC). All statistical analyses were conducted using SPSS version 14.0.

\section{Results}

Of the 54 subjects recruited for the study a total of 48 provided usable data. Four subjects were excluded because they did not provide information on the FSS at the follow up data collection period, 1 subject reported a major change in his life (new job), and 1 subject exceeded 4 weeks for the follow up data collection session.

Our sample was predominantly male $(\mathrm{n}=31,65 \%)$ with a mean age of 40.4 years $(\mathrm{SD}=12.6)$. There was a predominance of persons with tetraplegia $(n=26,54 \%)$ and ASIA A injuries $(\mathrm{n}=30,63 \%)$. The average duration since injury was 14.9 years $(\mathrm{SD}=11.8)$. The major cause of injury was motor vehicle accident $(n=27,56 \%)$. Seventy-five percent $(n=36)$ of our sample was unemployed and $25 \%(\mathrm{n}=12)$ employed or attending school.

The mean FSS score for the sample at baseline was $4.4(\mathrm{SD}=1.4)$ (Table 1). Just over $54 \%$ $(\mathrm{N}=26)$ of the subjects scored greater than 4 . The mean score was highest for item 1 ("motivation lower when fatigued") and lowest for item 2 ("exercise brings on fatigue").

The mean scores were 16.0 ( $\mathrm{SD}=10.8)$ on the CES-D; $5.3(\mathrm{SD}=2.8)$ on the VAS-F; and 52.7 $(\mathrm{SD}=18.1)$ on the SF-36 Vitality Score.

\section{Reliability}

The internal consistency of the FSS using Cronbach's alpha was 0.89. Item wise deletion altered the alpha coefficient by no more than 0.05 for any of the items. The two-week testretest reliability coefficient was ICC $=0.84$ with a $95 \% \mathrm{CI}$ of $0.74-0.90$. The item by item reliability ranged from a low of ICC $=0.32$ for item 1 to a high of 0.77 for item 5 (Table 1). The SEM for the FSS was 0.55 .

The Bland-Altman limits of agreement plot (Figure 2) demonstrates the absolute reliability of the FSS. The data points are well distributed along the 0 line and there are an equal 
number of points above and below the line suggesting minimal bias. None of the data points were found beyond 2 standard deviations (2.8) of the zero line.

\section{Validity}

Table 2 summarizes the correlations between the FSS and the other measures used in the study. The direction of the association for all measures was consistent with our hypotheses. The magnitude of the relationship was as hypothesized for the VAS- $\mathrm{F}(\mathrm{r}=.67)$ and symptoms of depression ( $\mathrm{r}=.58)$. However the magnitude of the relationship with the vitality sub scores $(r=-48)$ of the SF-36 was lower than hypothesized.

Assuming a FSS cut score of 4 to indicate significant fatigue and a VAS-F score of over 6 to indicate severe fatigue, we calculated the sensitivity of the FSS as $75 \%(18 / 24 ; 95 \%$ CI $55 \%$, $88 \%)$ and the specificity as $67 \%(16 / 24 ; 95 \%$ CI $50 \%, 84 \%)$. The diagnostic values for a cut score of score of 5 improved the specificity (83\% [95\%CI 64\%, 93\%]; 20/24) at the expense of sensitivity (54\% [95\% CI 35\%, 72\%];13/24). The area under the ROC was .799 indicating good ability to discriminate those with significant fatigue versus those without significant fatigue.

\section{DISCUSSION}

Clinical experience suggests fatigue can be an important problem in persons with SCI. However, accurate determination of the prevalence and magnitude of fatigue requires valid and reliable measures. We are unaware of any previous studies that addressed the validity and reliability of fatigue measures in persons with SCI.

The ideal approach to the evaluation of any clinical measure is comparison to a gold standard. There is no gold standard for the assessment of fatigue after SCI. In the absence of a gold standard, we chose to measure the reliability and validity of the FSS in a group of persons with ASIA A and ASIA B (motor complete) SCI. We chose the FSS because it is widely used, easy to administer, and has been well studied in other populations. $15,17,18,19,20,21,33$

Reliability is an essential attribute of any clinical or research measure. ${ }^{34}$ Internal consistency is an important component of reliability. The demonstrated internal consistency of the FSS in our study was as hypothesized. It was similar to that demonstrated previously in MS and SLE, myotonic dystrophy, and obesity. ${ }^{15,19,35}$ Higher values have been reported in polyneuropathies, chronic hepatitis $\mathrm{C}$, cancer, and renal failure. $5,18,20,36$ The alpha value obtained for our sample falls into the top or most desirable level based on Andresen's criteria but is below the threshold that some suggest indicates redundancy within the items. 34,37

Test-retest reliability is another important attribute of reliability. A variety of retest reliability values for the FSS have been reported in the literature ranging from ICC $=.82$ for individuals with chronic hepatitis $\mathrm{C}$ to ICC $=.88$ for individuals with myotonic dystrophy. ${ }^{18,35}$ The two week test-retest reliability for our study fell in the middle of this range (ICC $=.84)$ This level of reliability exceeds Andresen's standards. ${ }^{34}$ However some authors suggest that while 
ICC $=.80$ is acceptable for research, clinical use of a tool demands even less measurement error. $^{37}$

The individual items in the FSS were originally developed and validated for use in MS, raising the possibility that some of the items might not be appropriate for persons with SCI. We were therefore interested in the performance of the individual items on the nine item FSS. Analysis of item by item reliability ranged from a low of ICC $=0.32$ for item 1 to a high of 0.77 for item 5 . The sample mean score was highest for item 1 (motivation lower when fatigued) and lowest for item 2 (exercise brings on fatigue). Item 2 is of particular relevance to MS, in which symptoms may be exacerbated by exercise. However, it is likely of less relevance to fatigue in persons with SCI. Many of our subjects would have reduced capacity to exercise due to their motor impairment and level of lesion. It could be argued exclusion of Item 2 may strengthen the utility of the FSS in persons with SCI. However, item wise deletion altered the alpha coefficient by no more than 0.04 for any of the items. Modifying the FSS in persons with SCI would have the significant disadvantage of making it more difficult to compare fatigue in SCI to fatigue in other neurological impairments.

We used the Bland-Altman method to further assess reliability (Figure 2).Given that none of the data points were outside two standard deviations from the zero line we conclude that minimal bias exists within retest comparison of our sample. The scores were also observed to be well dispersed along the zero line and no one in our study scored at either extreme, supporting minimal floor or ceiling effect. Floor and ceiling effects are generally noted if at least $20 \%$ of the population score at either the top or bottom of the scale. ${ }^{34}$

Validity is another key attribute of a clinical or research measure. Assessment of validity in the absence of a gold standard is a common problem in rehabilitation measurement. ${ }^{34}$ In the absence of a gold standard, we addressed the question of validity by comparison to other measures relevant to the issue of fatigue in SCI. We chose measures shown to have acceptable validity in other settings that we felt were also relevant to persons with SCI. One such measure is the VAS-F. The VAS-F is simple, easy to administer, and has been widely used in other populations. We demonstrated a significant correlation between the VAS-F and the FSS, providing support for the validity of the FSS in SCI.

Depression is common after SCI ${ }^{10,11}$ and can contribute to fatigue. Based on the known overlap between symptoms of depression and fatigue, we compared the FSS to a well established measure of depressive symptoms, the CES-D. As hypothesized, we demonstrated a moderate but statistically significant correlation between the CES-D and the FSS. Interestingly, Krupp and colleagues also used the CES-D in assessing the relationship between fatigue and depression. ${ }^{15}$ The magnitude of their correlations were considerably smaller than ours for healthy controls $(r=.2)$ and individuals with MS ( $r=.26)$ while the sample with SLE was closer $(r=.46)$ to the association we obtained. Accounting for the difference in the magnitude between the samples is not straightforward. At least four of the items on the CES-D provide indirect reference to fatigue and therefore one would suspect a relatively strong relationship. Further research is needed to determine the relationship between depression and fatigue in SCI and whether fatigue measures like the FSS can distinguish depression from fatigue. 
We hypothesized there would be significant negative correlation between the vitality component of the SF-36 and the FSS. The magnitude of the correlation between the SF-36 Vitality Scale and the FSS in our study was less than expected. That may reflect the previously reported limitations of the SF-36 in SCI. ${ }^{24}$ Alternatively, the smaller than expected association may reflect the fact that vitality as captured by the SF-36 is a different construct than fatigue. Instruments developed specifically for the assessment of fatigue may be better tools for measuring fatigue in SCI than those that are part of a larger testing battery.

Over 50\% of our subjects had FSS scores greater than 4, a score that has been reported to indicate clinically significant fatigue. Using a cut score of over 4 yielded better sensitivity than a cut score of over 5, but specificity was better for an FSS score of over 5. Near perfect sensitivity (96\%) is observed if a cut score of over 3 is selected. Choosing the best cut score for a tool depends on the diagnostic goal and any associated stigma associated with having the condition. ${ }^{38}$ Put another way, high sensitivity of a test is preferred if having the "disease" is serious and/or the "cure" is relatively inexpensive and easily available. Severe fatigue is not life threatening but may be associated with personal costs (activity limitations and participation restrictions), societal costs (increased burden of care and reduced employability), and health care costs (greater risk for medical complications and visits to health care providers).

It is tempting to reach conclusions about the prevalence and severity of fatigue in persons with SCI based on our results, but that was not the purpose of our study. The cut score for the FSS influences the prevalence for significant fatigue in our study population, which ranges from $35 \%$ for a score of over 5 to $56 \%$ for a score of over 4 . The generalizability of this threshold warrants additional study as others have found that cut points for other tools such as the CES-D do not necessarily transfer to the SCI population. ${ }^{26}$

Our study does have some limitations. Our sample included only healthy volunteers with motor complete SCI. We chose to recruit from this sub-group to avoid the potential confounding effects of including subjects with a wide range of neurological impairments and health conditions. We had a relatively high number of persons with tetraplegia in our study, and it is possible that could have influenced our results.

Based on clinical experience, fatigue is a problem in those with both complete and incomplete motor SCI. We can not be certain our results will apply to persons with motor incomplete SCI, but we have no reason to believe that will not be the case. Further research is required to address that issue and relationship of lesion level and completeness to fatigue. Finally, our study did not test the responsiveness or predictive validity of the FSS. We plan to conduct additional analysis and research to further evaluate the psychometric shortcomings of the current study.

\section{CONCLUSIONS}

Fatigue is a well described problem after SCI that may increase disability and reduce quality of life. The FSS is a widely used fatigue measure previously shown to have acceptable validity in a wide range of populations. Our study has demonstrated the FSS has acceptable 
reliability with regard to internal consistency, test-retest reliability, and validity for use in persons with SCI. Our study also provides support for the idea that fatigue is an important clinical problem in persons with SCI.

\section{Acknowledgments}

Funding for this project was provided by the Rick Hansen Man in Motion Foundation Research Fund. Dr. Miller receives salary support from the Canadian Institutes of Health Research (CIHR \#76731). Preliminary data from this study was presented at the combined annual meetings of the American Spinal Injury Association (ASIA) and the International Spinal Cord Society (ISCoS). No commercial party having a direct financial interest in the results of this research has or will confer a benefit upon the authors or any organization with which the authors are associated.

\section{References}

1. Chaudhuri A, Behan OP. Fatigue in neurologic disorders. Lancet. 2004; 363:978-88. [PubMed: 15043967]

2. Freal JG, Kraft GH, Coryell JK. Symptomatic fatigue in multiple sclerosis. Arch Phys Med Rehabil. 1984; 5:135-37.

3. Ingles JL, Eskes GA, Phillips SJ. Fatigue after stroke. Arch Phys Med Rehabil. 1999; 80:173-178. [PubMed: 10025492]

4. Merkies ISJ, Schmitz PIM, Samijn JPA, van der Meche FGA, van Doorn PA. Fatigue in immunemediated polyneuropathies. Neurology. 1999; 53:1648-1654. [PubMed: 10563607]

5. Vasconcelos OM Jr, Prokhorenko OA, Kelley KF, Vo AH, Olsen CH, Dalakas MC, Halstead LS, Jabbari B, Campbell WW. A comparison of fatigue scales in postpoliomyelitis syndrome. Arch Phys Med Rehabil. 2006; 87:1213-17. [PubMed: 16935057]

6. McColl MA, Arnold R, Charlifue S, Glass C, Savic G, Frankel H. Aging, spinal cord injury, and quality of life: structural relationships. Arch Phys Med Rehabil. 2003; 84:1137-44. [PubMed: 12917851]

7. Gerhart KA, Bergstrom E, Charlifue SW, Menter RR, Whiteneck GG. Long-term spinal cord injury: functional changes over time. Arch Phys Med Rehabil. 1993; 74:1030-4. [PubMed: 8215852]

8. Charlifue SW, Weitzenkamp DA, Whiteneck GG. Longitudinal outcomes in spinal cord injury: aging, secondary conditions, and well-being. Arch Phys Med Rehabil. 1999; 80:1429-34. [PubMed: 10569437]

9. Hirsch GH, Menard MM, Anton HA. Anemia in Spinal Cord Injury. Arch Phys Med Rehabil. 1991; 72:195-201. [PubMed: 1998453]

10. Elliott TR. Depression following spinal cord injury. Arch Phys Med Rehabil. 1996; 77:816-23. [PubMed: 8702378]

11. Hughes R, Swedlund N, Petersen N, Nosek MA. Depression and Women with Spinal Cord Injury. Topics in SCI. Rehabilitation. 2001; 7:16-24.

12. Streeten DH, Anderson GH. The role of delayed orthostatic hypotension in the pathogenesis of chronic fatigue. Clin Autonomic Research. 1998; 8:119-124.

13. Widerstrom-Noga EG, Felipe-Cuervo E, Yezierski RP. Chronic pain after spinal cord injury: Interference with sleep and daily activities. Arch Phys Med Rehabil. 2001; 82:1571-1577. [PubMed: 11689978]

14. Weingarden SI, Martin C. Independent dressing after spinal cord injury: a functional time evaluation. Arch Phys Med Rehabil. 1989; 70:518-9. [PubMed: 2742466]

15. Krupp LB, LaRocca NG, Muir-Nash J, Steinberg AD. The Fatigue Severity Scale: Application to patents with multiple sclerosis and systemic lupus erythematosus. Arch Neurol. 1989; 46:1121-3. [PubMed: 2803071]

16. Dittner AJ, Wessely SC, Brown RG. The assessment of fatigue: A practical guide for clinicians and researchers. Journal of Psychosomatic Research. 2004; 56:157-170. [PubMed: 15016573]

17. Herlofson K, Larsen JP. Measuring fatigue in patients with Parkinson's disease - the Fatigue Severity Scale. European Journal of Neurology. 2002; 9:595-600. [PubMed: 12453074] 
18. Kleinman L, Zodet MW, Hakim Z, Aledort J, Barker C, Chan K, Krupp L, et al. Psychometric evaluation of the fatigue severity scale for use in chronic hepatitis C. Quality of Life Research. 2000; 9:499-508. [PubMed: 11190005]

19. Grieve FG, Harris DS, Fairbanks SD. Extending the Fatigue Severity Scale to an obese population. Eating Weight Disord. 2000; 5:161-165.

20. Winstead-Fry P. Psychometric assessment of four fatigue scales with a sample of rural cancer patients. J Nursing Meas. 1998; 6:111-22.

21. Schepers VP, Visser-Meily AM, Ketelaar M, et al. Poststroke Fatigue: Course and its relation to personal and stroke-related factors. Arch Phys Med Rehabil. 2006; 87:184-88. [PubMed: 16442970]

22. ASIA. International Standards for the Neurological Classification of Spinal Cord Injury. 2002. (revised 2002)

23. Ware JJ, Sherbourne CD. The MOS 36-item short-form health survey (SF-36). I. Conceptual framework and item selection. Medical Care. 1992; 30:473-83. [PubMed: 1593914]

24. Forchheimer M, McAweeney M, Tate DG. Use of the SF-36 among persons with spinal cord injury. Am J Phys Med Rehabil. 2004; 83:390-395. [PubMed: 15100631]

25. Radloff LS. The CES-D scale: a self-report depression scale for research in the general population. Appl Psychol Meas. 1977; 1:385-401.

26. Kuptniratsaikul V, Chulakadabba S, Ratanavijitrasil S. An instrument for assessment of depression among spinal cord injury patients: Comparison between the CES-D and TDI. J Med Assoc Thai. 2002; 85:978-83. [PubMed: 12450075]

27. Kos D, Nagels G, D'Hooghe MB, et al. A rapid screening tool for fatigue impact in multiple sclerosis. BMC Neurol. 2006; 6:27. Published online 2006 August 17. [PubMed: 16916440]

28. LaChapelle D, Alfano D. Revised neurobehavioral scales of the MMPI: Sensitivity and specificity in traumatic brain injury. Appl Neuropsychol. 2005; 12:143-150. [PubMed: 16131341]

29. Donner A, Eliazew M. Sample size requirements for reliability studies. Statistics in Medicine. 1987; 4:441-448.

30. Portney, LG., Watkins, MP. Foundations of Clinical Research: Applications to Practice. Upper Saddle River, NJ: Prentice Hall Health; 2000.

31. Bland JM, Altman DG. The statistical method for assessing agreement between two methods of clinical measurement. Lancet. 1986; 1:307-310. [PubMed: 2868172]

32. Rankin G, Stokes M. Reliability of assessment tools in rehabilitation: an illustration of appropriate statistical analyses. Clin Rehabil. 1998; 12:187-199. [PubMed: 9688034]

33. LaChapelle DL, Finlayson MAJ. An evaluation of subjective and objective measures of fatigue in patients with brain injury and healthy controls. Brain Injury. 1998; 12:649-59. [PubMed: 9724836]

34. Andresen EM. Criteria for assessing the tools of disability outcomes research. Arch Phys Med Rehabil. 2000; 81S2:S15-20.

35. Laberge L, Gagnon C, Jean S, Mathieu J. Fatigue and daytime sleepiness rating scales in myotonic dystrophy: A study of reliability. J Neurol Neurosurg Psychiatry. 2005; 76:1403-5. [PubMed: 16170085]

36. Schneider RA. Chronic renal failure: Assessing the fatigue severity scale for use among caregivers. J Clin Nurs. 2004; 13:219-225. [PubMed: 14723674]

37. Streiner, DL., Norman, JR. Health measurement scales: a practical guide to their development and use. New York, NY: Oxford University Press; 2003.

38. Sackett, DL. Clinical epidemiology: a basic science for medicine. 2. Boston: Little Brown; 1991. 


\begin{tabular}{|c|c|c|c|c|c|c|c|}
\hline \multirow{2}{*}{$\begin{array}{l}\text { During the past week, I have found that: } \\
\text { 1. My motivation is lower when I am fatigued. }\end{array}$} & \multicolumn{7}{|c|}{ Score } \\
\hline & 1 & 2 & 3 & 4 & 5 & 6 & 7 \\
\hline 2. Exercise brings on my fatigue. & 1 & 2 & 3 & 4 & 5 & 6 & 7 \\
\hline 3. I am easily fatigued. & 1 & 2 & 3 & 4 & 5 & 6 & 7 \\
\hline 4. Fatigue interferes with my physical functioning. & 1 & 2 & 3 & 4 & 5 & 6 & 7 \\
\hline 5. Fatigue causes frequent problems for me. & 1 & 2 & 3 & 4 & 5 & 6 & 7 \\
\hline 6. My fatigue prevents sustained physical functioning. & 1 & 2 & 3 & 4 & 5 & 6 & 7 \\
\hline 7. Fatigue interferes with carrying out certain duties and responsibilities. & 1 & 2 & 3 & 4 & 5 & 6 & 7 \\
\hline 8. Fatigue is among my three most disabling symptoms. & 1 & 2 & 3 & 4 & 5 & 6 & 7 \\
\hline 9. Fatigue interferes with my work, family, or social life. & 1 & 2 & 3 & 4 & 5 & 6 & 7 \\
\hline
\end{tabular}

Choose a number from 1 to 7 that indicates your degree of agreement with each statement. $1=$ strongly disagree and $7=$ strongly agree.

Figure 1.

FATIGUE SEVERITY SCALE QUESTIONNAIRE 


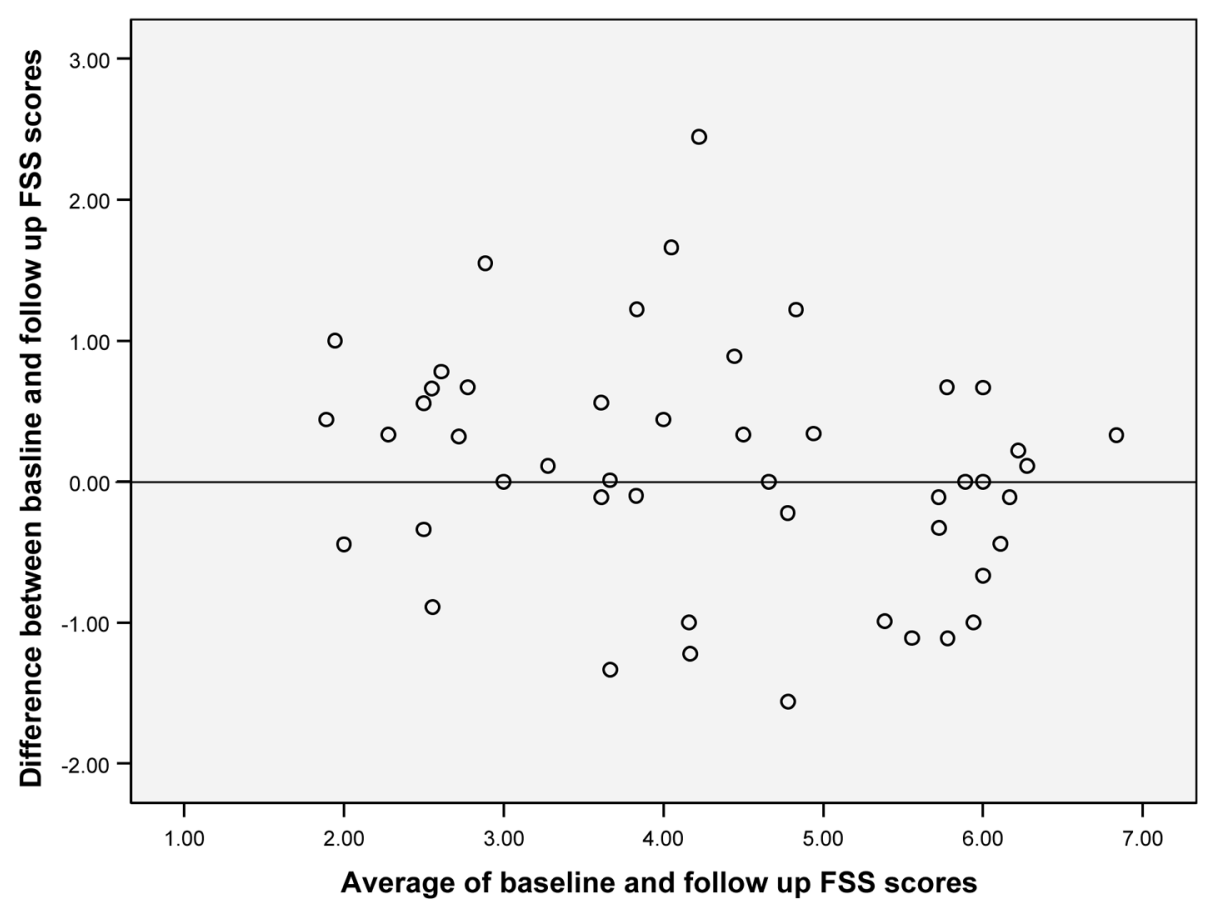

Figure 2.

Bland Altman Plot of FSS Difference and Average Scores 


\section{Table 1}

Mean and Intraclass Correlation Coefficient (ICC) Values for Total and Individual Fatigue Severity Scale Items

\begin{tabular}{clll}
\hline Item Number & Mean (SD) & ICC & CI 95\% \\
\hline 1 & $5.7(1.6)$ & 0.32 & $0.03-0.56$ \\
2 & $3.8(1.7)$ & 0.42 & $0.14-0.63$ \\
3 & $4.1(1.8)$ & 0.51 & $0.26-0.70$ \\
4 & $4.7(1.9)$ & 0.73 & $0.56-0.85$ \\
5 & $4.0(1.7)$ & 0.77 & $0.62-0.87$ \\
6 & $4.1(1.9)$ & 0.74 & $0.58-0.85$ \\
7 & $4.2(1.9)$ & 0.70 & $0.51-0.82$ \\
8 & $4.1(2.0)$ & 0.75 & $0.58-0.85$ \\
9 & $4.3(2.1)$ & 0.74 & $0.58-0.85$ \\
Total & $4.4(1.4)$ & 0.84 & $0.74-0.90$ \\
\hline
\end{tabular}




\section{Table 2}

Correlation between FSS and Other Study Measures

\begin{tabular}{|lrl|}
\hline Variables & $\mathbf{r}$ & $\mathbf{p}$ Value \\
\hline Fatigue VAS & 0.67 & .000 \\
CES-D & 0.58 & .001 \\
SF-36 Vitality Score & -0.48 & .010 \\
\hline
\end{tabular}

